\title{
Transcriptional regulation of thylakoid galactolipid biosynthesis coordinated with chlorophyll biosynthesis during the development of chloroplasts in Arabidopsis
}

\author{
Koichi Kobayashi ${ }^{1}$, Sho Fujii ${ }^{1}$, Daichi Sasaki ${ }^{1}$, Shinsuke Baba ${ }^{2}$, Hiroyuki Ohta ${ }^{2,3,4}$, Tatsuru Masuda ${ }^{1}$ and \\ Hajime Wada ${ }^{1,4}$
}

${ }^{1}$ Graduate School of Arts and Sciences, The University of Tokyo, Tokyo, Japan

${ }^{2}$ Center for Biological Resources and Informatics, Tokyo Institute of Technology, Yokohama, Japan

${ }^{3}$ Earth-Life Science Institute, Tokyo Institute of Technology, Tokyo, Japan

${ }^{4}$ Core Research for Evolutional Science and Technology, Japan Science and Technology Agency, Tokyo, Japan

\section{Edited by:}

Bernd Mueller-Roeber, University of

Potsdam, Germany

\section{Reviewed by:}

Salma Balazadeh, University of Potsdam, Germany

Federico Valverde, Instituto de Bioquímica Vegetal y Fotosíntesis -

Consejo Superior de Investigaciones Científicas, Spain

\section{${ }^{*}$ Correspondence:}

Koichi Kobayashi, Graduate School of Arts and Sciences, The University of Tokyo, 3-8-1 Komaba, Meguro-ku, Tokyo 153-8902, Japan e-mail: kkobayashi@bio.c.u-tokyo.ac.jp
Biogenesis of thylakoid membranes in chloroplasts requires the coordinated synthesis of chlorophyll and photosynthetic proteins with the galactolipids monogalactosyldiacylglycerol (MGDG) and digalactosyldiacylglycerol (DGDG), which constitute the bulk of the thylakoid lipid matrix. MGD1 and DGD1 are the key enzymes of MGDG and DGDG synthesis, respectively. We investigated the expression profiles of MGD1 and DGD1 in Arabidopsis to identify the transcriptional regulation that coordinates galactolipid synthesis with the synthesis of chlorophyll and photosynthetic proteins during chloroplast biogenesis. The expression of both MGD1 and DGD1 was repressed in response to defects in chlorophyll synthesis. Moreover, these genes were downregulated by norflurazon-induced chloroplast malfunction via the GENOMES-UNCOUPLED1-mediated plastid signaling pathway. Similar to other photosynthesis-associated nuclear genes, the expression of MGD1 and DGD1 was induced by light, in which both cytokinin signaling and LONG HYPOCOTYL5-mediated light signaling played crucial roles. The expression of these galactolipid-synthesis genes, and particularly that of DGD1 under continuous light, was strongly affected by the activities of the GOLDEN2-LIKE transcription factors, which are potent regulators of chlorophyll synthesis and chloroplast biogenesis. These results suggest tight transcriptional coordination of galactolipid synthesis with the formation of the photosynthetic chlorophyllprotein complexes during leaf development. Meanwhile, unlike the photosynthetic genes, the galactolipid synthesis genes were not upregulated during chloroplast biogenesis in the roots, even though the galactolipids accumulated with chlorophylls, indicating the importance of post-transcriptional regulation of galactolipid synthesis during root greening. Our data suggest that plants utilize complex regulatory mechanisms to modify galactolipid synthesis with chloroplast development during plant growth.

Keywords: chlorophyll, chloroplast development, digalactosyldiacylglycerol, galactolipid, monogalactosyldiacylglycerol, photosynthesis, transcriptional regulation

\section{INTRODUCTION}

Autotrophic plant growth depends on photosynthesis, which takes place in the photosynthetic plastids, i.e., the chloroplasts. Whereas some core photosynthetic proteins are encoded in the plant plastid genome, many photosynthetic proteins, such as light-harvesting complex subunits, are encoded in the nuclear genome and targeted to chloroplasts after synthesis in the cytosol (Jarvis and López-Juez, 2013). All the genes involved in chlorophyll synthesis also reside in the angiosperm nuclear genome (Tanaka et al., 2011). At the onset of chloroplast biogenesis, enzymes involved in chlorophyll synthesis are actively expressed together with nuclearencoded photosynthetic proteins, and are then transported to the chloroplasts where chlorophyll synthesis takes place. Because chlorophylls and their intermediates are strong photosensitizers and are phototoxic, plant cells strictly regulate their metabolism to coincide with the development of the photosynthetic machinery.
In addition to modifications in enzymatic activities, transcriptional regulation plays a pivotal role in regulating chlorophyll synthesis with chloroplast functionality (Tanaka et al., 2011). When chloroplast function is severely impaired, the expression of chlorophyll synthesis genes is downregulated globally along with nuclear photosynthetic genes, through plastid-to-nucleus retrograde signaling (Strand et al., 2003; Moulin et al., 2008).

As light strongly stimulates chloroplast development, the light signaling pathway is heavily involved in transcriptional regulation of chlorophyll synthesis and formation of the photosynthetic machinery. Before light exposure, basic helixloop-helix transcription factors, named PHYTOCHROMEINTERACTING FACTORS (PIFs), repress genes involved in chlorophyll synthesis, whereas a basic Leu zipper transcription factor, LONG-HYPOCOTYL5 (HY5), upregulates these genes, together with nuclear photosynthetic genes, on illumination 
(Tanaka etal., 2011). Yet other types of transcription factors, such as GOLDEN2-LIKE1 (GLK1) and GLK2, participate in the regulation of chlorophyll synthesis genes and nuclear photosynthetic genes. Deficiencies of both GLK1 and GLK2 cause a notable reduction in the expression of chlorophyll synthesis genes in leaves (Fitter etal., 2002; Waters et al., 2009), whereas overexpression of these factors induces strong expression of the chlorophyll-related genes, with ectopic chlorophyll accumulation in non-photosynthetic organs (Nakamura et al., 2009; Kobayashi etal., 2012a; Powell etal., 2012), suggesting that GLK factors are potent upregulators of these target genes. Recently, the cytokinin-responsive GATA transcription factors GATA NITRATE-INDUCIBLE CARBON-METABOLISMINVOLVED (GNC) and CYTOKININ-RESPONSIVE GATA TRANSCRIPTION FACTOR1 (CGA1) were also reported to induce ectopic chlorophyll accumulation in hypocotyl epidermis upon overexpression (Chiang et al., 2012). In contrast to the direct regulation of the chlorophyll synthesis genes by HY5, PIFs, and GLKs, the GATA factors upregulate the genes in an indirect manner, and their regulatory pathways remain unclear (Hudson et al., 2011).

The photosynthetic chlorophyll-protein complexes are embedded in a lipid matrix of thylakoid membranes, mainly composed of the galactolipids monogalactosyldiacylglycerol (MGDG) and digalactosyldiacylglycerol (DGDG). These lipids are required not only for formation of the lipid bilayer but also for the structure and function of the photosynthetic complexes (Kobayashi et al., 2013a). Therefore, a coordinated synthesis of galactolipids with chlorophylls and photosynthetic proteins is essential for the construction of the thylakoid membrane networks. The synthesis of MGDG is catalyzed by MGDG synthase, which transfers galactose from UDP-galactose to diacylglycerol (Kobayashi et al., 2009b). Of the three MGDG synthases (MGD1, MGD2, and MGD3) in Arabidopsis, MGD1 is the major isoform responsible for the bulk of galactolipid synthesis (Kobayashi et al., 2007), whereas MGD2 and MGD3 constitute an alternative pathway, functioning under phosphate-deficient growth conditions (Kobayashi et al., 2009a). The expression of MGD1 is widespread in photosynthetic tissues, whereas those of MGD2 and MGD3 are rarely found in green tissues under nutrientsufficient conditions; however, MGD2 and MGD3 expression is strongly induced under phosphate deficient conditions (Awai et al., 2001; Kobayashi et al., 2004). Not only is MGDG the main constituent of thylakoid membranes, it is also a substrate for DGDG synthesis. DGDG is predominantly synthesized by the addition of a second galactose from UDP-galactose to MGDG, which is catalyzed by DGDG synthase (Kelly and Dörmann, 2002; Kelly etal., 2003). Higher plants, including Arabidopsis, also have paralogous genes for DGDG synthase, namely DGD1 and DGD2 (Gaude et al., 2004). Analysis of Arabidopsis mutants has shown that the bulk of DGDG found in chloroplasts is synthesized by DGD1 in combination with MGD1 (Dörmann et al., 1995).

Recently we reported that a defect in galactolipid synthesis leads to strong downregulation of chlorophyll synthesis and photosynthetic genes, suggesting a coupled regulation between galactolipid synthesis and the formation of photosynthetic chlorophyll-protein complexes (Kobayashi et al., 2013a). Considering that galactolipids are essential components of thylakoid membranes and photosynthesis, their synthesis is expected to be regulated alongside that of chlorophylls and photosynthetic proteins. It has been reported that the activity of MGD1 is post-translationally controlled by a redox-dependent oxidation/reduction (Yamaryo et al., 2006; Shimojima et al., 2013) and by dissociation/association of phosphatidic acid (Dubots et al., 2010; Shimojima et al., 2013). On the other hand, there is only limited data on the transcriptional regulation of galactolipid synthesis during chloroplast biogenesis (Kobayashi et al., 2009b), in contrast to the in-depth insights into the regulatory mechanisms of the genes involved in chlorophyll synthesis and photosynthesis (Tanaka et al., 2011; Jarvis and López-Juez, 2013). In this study, we investigated the relationships among the expression of galactolipid synthesis genes and chlorophyll synthesis, chloroplast functionality, light and cytokinin signaling, and several chloroplast-related transcription factors.

\section{MATERIALS AND METHODS PLANT MATERIALS AND GROWTH CONDITIONS}

All plants used in this study were the Columbia ecotype of Arabidopsis thaliana. The cs (Koncz et al., 1990), chli1, chlh (Huang and Li, 2009), hema1 (Woodson et al., 2011), genomes-uncoupled (gun) 1-1, pOCA107-2 (the parental line for the gun mutants; Susek et al., 1993), gun4-1, gun5-1 (Mochizuki et al., 2001), hy5215 (Oyama et al., 1997), ahk2-2 ahk3-3 (Higuchi et al., 2004), glk1 glk2 (Fitter et al., 2002), GLK1ox (35S:GLK1), GLK2ox (35S:GLK2; Waters etal., 2008), gnc cgal (Chiang etal., 2012), and slr-1 (Fukaki etal., 2002) mutants were described previously. Plants were grown on Murashige and Skoog (MS) medium (adjusted to $\mathrm{pH} 5.7$ with $\mathrm{KOH}$ ) containing $1.0 \%(\mathrm{w} / \mathrm{v})$ sucrose solidified with $0.8 \%(\mathrm{w} / \mathrm{v})$ agar in plates at $23^{\circ} \mathrm{C}$ under continuous white light $\left(40 \mu \mathrm{mol}\right.$ photons $\mathrm{m}^{-2} \mathrm{~s}^{-1}$ ), with the exception of the experiment in Figure 3. For the analysis in Figure 3, sterilized seeds were sown in flasks with $20 \mathrm{~mL}$ liquid MS media. Each flask was completely wrapped with aluminum foil after $3 \mathrm{~h}$ of light exposure and incubated on the rotary shaker at $22^{\circ} \mathrm{C}$. After growth for 4 days in the dark, etiolated seedlings were treated with white light $\left(40 \mu \mathrm{mol}\right.$ photons $\mathrm{m}^{-2} \mathrm{~s}^{-1}$ ) or with $1 \mu \mathrm{M}$ 6-benzyladenine (BA) for the indicated period. For the norflurazon (NF) treatment in Figure 2, plants were germinated and grown in MS medium containing $1 \mu \mathrm{M}$ NF. For the analysis of roots, plants were grown vertically in the MS medium for the indicated period, and roots were detached from the shoot at the root-hypocotyl junction for sampling. For the BA treatment in Figure 5A, 7-day-old wild-type seedlings were transferred to the MS medium containing $1 \mu \mathrm{M}$ BA and grown for another 7 days. All plants were grown in a growth chamber (CLE-303, Tomy Seiko, Tokyo, Japan).

\section{CHLOROPHYLL DETERMINATION}

Whole seedlings were pulverized in liquid nitrogen, homogenized in $80 \%(\mathrm{v} / \mathrm{v})$ acetone, and debris was removed by centrifugation at $10,000 \times g$ for $5 \mathrm{~min}$. The absorbances of the supernatant at $720 \mathrm{~nm}, 663 \mathrm{~nm}, 647 \mathrm{~nm}$, and $645 \mathrm{~nm}$ were measured using an Ultrospec 2100 pro spectrophotometer (GE Healthcare Bioscience, 
Amersham, UK). The chlorophyll ( $a$ and $b$ ) concentration of the samples was determined as described in Melis et al. (1987).

\section{GENE EXPRESSION ANALYSIS}

Gene expression was examined by a real-time quantitative RT-PCR (Q-PCR) analysis (Pfaffl, 2001). Total RNA was extracted using the RNeasy Plant Mini kit (Qiagen, Hilden, Germany). Genomic DNA digestion and reverse transcription were performed using the PrimeScript RT Reagent kit with gDNA Eraser (TaKaRa Bio, Otsu, Japan) according to the manufacturer's instructions. cDNA amplification was performed using the Thunderbird PreMix kit (Toyobo, Osaka, Japan) and $200 \mathrm{nM}$ of the following genespecific primers: MGD1 (AT4G31780): forward primer, 5' GCAGGACTTGAAACATCACAAATC-3'; reverse primer, $5^{\prime}$ GCGAACTGGTTTCACAAAGGA-3'; DGD1 (AT3G11670): forward primer, 5'-CTGAAGAGAGATCCCGTGGTG-3'; reverse primer, 5' -TCCCAAGTTCGCTTTTGTGTT-3'; DGD2 (AT4G005 50): forward primer, 5'-TGCAGAACCTATGACGATGGA-3' reverse primer, 5' -GCTCTGTAAGTTGCGATGGTTG-3'; LIGHT HARVESTING COMPLEX PHOTOSYSTEM II SUBUNIT 6 (LHCB6, AT1G15820): forward primer, 5'-GGACTTTGAGAAGCTGGAGAGG-3'; reverse primer, 5' -ACAAACCAAGAGCACCGAGAG-3'; H SUBUNIT OF MG-CHELATASE (CHLH, AT5G13630): forward primer, 5' -TGGTAGAGAGACAGAAGCTCGAAA- $3^{\prime}$; reverse primer, 5' ${ }^{\prime}$ CCAAAGAACCTGCCCAAGAG-3'; ACTIN8 (AT1G49240): forward primer, 5'-ACTGTGCCTATCTACGAGGGTTTC-3'; reverse primer, 5' -CCCGTTCTGCTGTTGTGGT-3'. The amplification efficiency with each primer set was determined with a dilution series of the cDNA pool from wild-type samples. No or negligible signals were detected in notemplate control with each primer set. Thermal cycling consisted of an initial denaturation step at $95^{\circ} \mathrm{C}$ for $10 \mathrm{~s}$, followed by 40 cycles of $95^{\circ} \mathrm{C}$ for $5 \mathrm{~s}$ and $60^{\circ} \mathrm{C}$ for $30 \mathrm{~s}$. Melting curve runs were performed at the end of each PCR to verify the specificity of the primers. Signal detection and quantification were performed in duplicate using a MiniOpticon (Bio-Rad, Hercules, CA, USA). The relative abundance of all transcripts amplified was normalized to the constitutive expression level of ACTIN8 according to the efficiency correction method (Pfaffl, 2001). Three independent biological experiments were performed for each sample.

\section{LIPID AND FATTY ACID ANALYSES}

Total lipids were extracted from roots pulverized in liquid nitrogen and were separated by thin-layer chromatography using a solvent system of acetone/toluene/water (136:45:12, v/v/v) as described by Dörmann et al. (1995) and Yamaryo et al. (2003). Lipids were visualized with $0.01 \%(\mathrm{w} / \mathrm{v})$ primuline in $80 \%(\mathrm{v} / \mathrm{v})$ acetone under UV light. MGDG, DGDG, and a mixture of other glycerolipids, including phosphatidylcholine, phosphatidylethanolamine, phosphatidylinositol, phosphatidylglycerol, and sulfoquinovosyldiacylglycerol, were isolated from silica gel plates. Fatty acids in each lipid fraction were methyl-esterified by incubating with a $5 \%(\mathrm{v} / \mathrm{v})$ $\mathrm{HCl}$ in methanol solution at $85^{\circ} \mathrm{C}$ for $2 \mathrm{~h}$ and quantified by gas chromatography (GC-17, Shimadzu, Kyoto, Japan) using myristic acid as an internal standard.

\section{STATISTICAL ANALYSIS}

Two-tailed Student's $t$-test with equal variance was performed using Microsoft Excel 2010 for Windows.

\section{RESULTS \\ THE INFLUENCE OF CHLOROPHYLL SYNTHESIS ON MGD1 AND DGD1 EXPRESSION}

To address whether chlorophyll metabolism influences expression of galactolipid synthesis genes, we examined the expression levels of MGD1 and DGD1 in chlorophyll-deficient mutants. chlh, chli1 (Huang and Li, 2009), and hemal (Woodson et al., 2011) are knockout mutants for CHLH, CHLI1, and HEMA1, respectively, whereas the cs mutant (Koncz et al., 1990) is a knockdown mutant for CHLI1. HEMA1 encodes the major isoform of glutamyl-tRNA reductase, catalyzing the synthesis of 5-aminolevulinic acid (the first committed step of tetrapyrrole synthesis; Tanaka et al., 2011). $\mathrm{CHLH}$ is a single gene encoding the subunit $\mathrm{H}$ of magnesium chelatase, whereas CHLI1 encodes the major isoform of the two paralogs of magnesium chelatase subunit I in Arabidopsis. We compared chlorophyll contents in 7-day-old seedlings among the mutants and wild-type seedlings (Figure 1A). As reported previously, the chlh mutant did not contain any chlorophyll (Huang and Li, 2009), whereas the cs mutant contained half the level of chlorophyll present in the wild-type (Rissler et al., 2002; Kobayashi et al., 2008). The hemal and chli1 mutants had 95\% less chlorophyll than the wild-type seedlings, consistent with previous reports (Huang and Li, 2009; Kobayashi et al., 2013a). Q-PCR analyses revealed that the chlh mutant had the largest decrease in MGD1 and DGD1

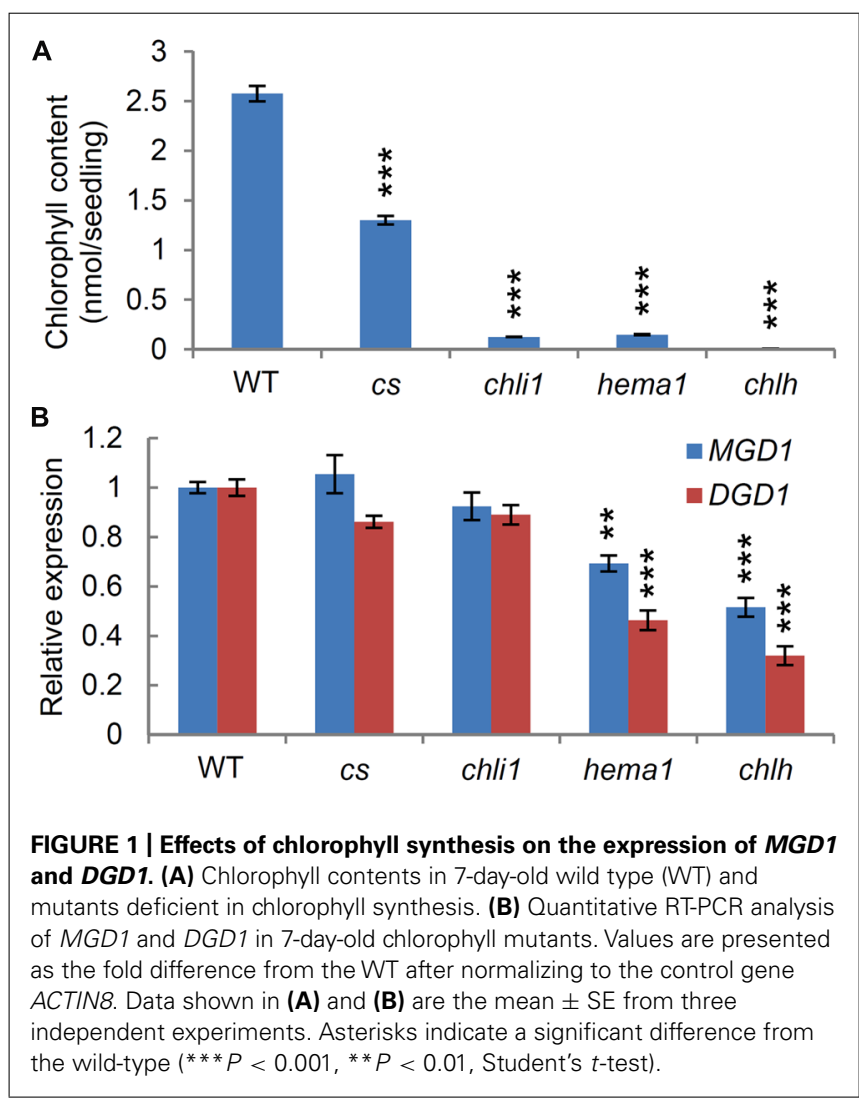


expression, suggesting that a complete loss of chlorophyll synthesis induces downregulation of the major galactolipid synthesis genes. There was also a significant decrease in MGD1 and DGD1 expression in the hema1 mutant. Although total chlorophyll levels were equivalent in chlil and hemal mutants, the expression levels of MGD1 and DGD1 in chli1 were higher than those in hema1, and were almost comparable to the wild-type. In addition, no significant differences in the expression levels of the galactolipid synthesis genes were observed between the wild-type and the cs mutant. As HEMA1 operates within a pathway that is also used for tetrapyrrole, heme, and chlorophyll synthesis (Tanaka et al., 2011), the hemal mutation could have a greater effect than the chil1 mutation, which might result in stronger repression of $M G D 1$ and DGD1. Our data suggest that it is not simply the level of chlorophyll synthesis that influences the expression of the major galactolipid synthesis genes, but also the functional state of tetrapyrrole and/or chloroplast metabolism.

\section{THE INVOLVEMENT OF RETROGRADE SIGNALING IN MGD1 AND DGD1 EXPRESSION}

The reduced expression of MGD1 and DGD1 in chlh and hemal suggests a repression mechanism of these genes in response to an impairment of tetrapyrrole synthesis or chloroplast function. It is known that photosynthesis-associated nuclear genes are downregulated through signals transmitted retrogradely from plastids (plastid signals) when chloroplast functions are severely disrupted (Tanaka et al., 2011; Jarvis and López-Juez, 2013). To assess whether plastid signals are involved in the regulation of galactolipid synthesis genes, we examined expression levels of MGD1 and DGD1 in gun mutants (gun1, gun4, and gun5), which are deficient in plastid signaling (Susek et al., 1993; Mochizuki etal., 2001). The pOCA107-2 line, which is the parental line of the gun mutants (Susek et al., 1993), was used as the wild-type control.

Plants were germinated and grown for 3 days (Figure 2A, left) or 4 days (Figure $2 \mathrm{~A}$, right) in the presence or absence of NF, which inhibits carotenoid synthesis and, consequently, chloroplast functions. As reported for many other photosynthesis-associated nuclear genes (Strand et al., 2003), expression levels of LHCB6 and CHLH were substantially decreased by NF treatment in the wildtype seedlings (Figure 2A). MGD1 and DGD1 expression also decreased in the 3-day-old wild type after NF treatment, although not as much as $L H C B 6$ and $C H L H$. In the 4-day-old wild type, $M G D 1$ and $D G D 1$ expression was more strongly repressed than in the 3-day-old wild-type, suggesting that a longer NF treatment led to stronger downregulation of these genes. In the gun 1 mutant, expression levels of all these genes did not differ from those in the wild-type in the absence of NF. Meanwhile, LHCB6 and $C H L H$ expression in the NF-treated gun 1 mutant was higher than that in the NF-treated wild type, consistent with previous reports (Susek et al., 1993; Moulin et al., 2008). Moreover, the 3day-old gun1 seedlings showed no obvious decrease in MGD1 and $D G D 1$ expression upon NF treatment. Even in the 4-day-old gun 1 seedlings, higher expression of MGD1 and DGD1 was observed compared with that in wild-type. These results suggest that GUN1 plays a pivotal role in the downregulation of the major galactolipid synthesis genes when chloroplast functions are severely impaired.

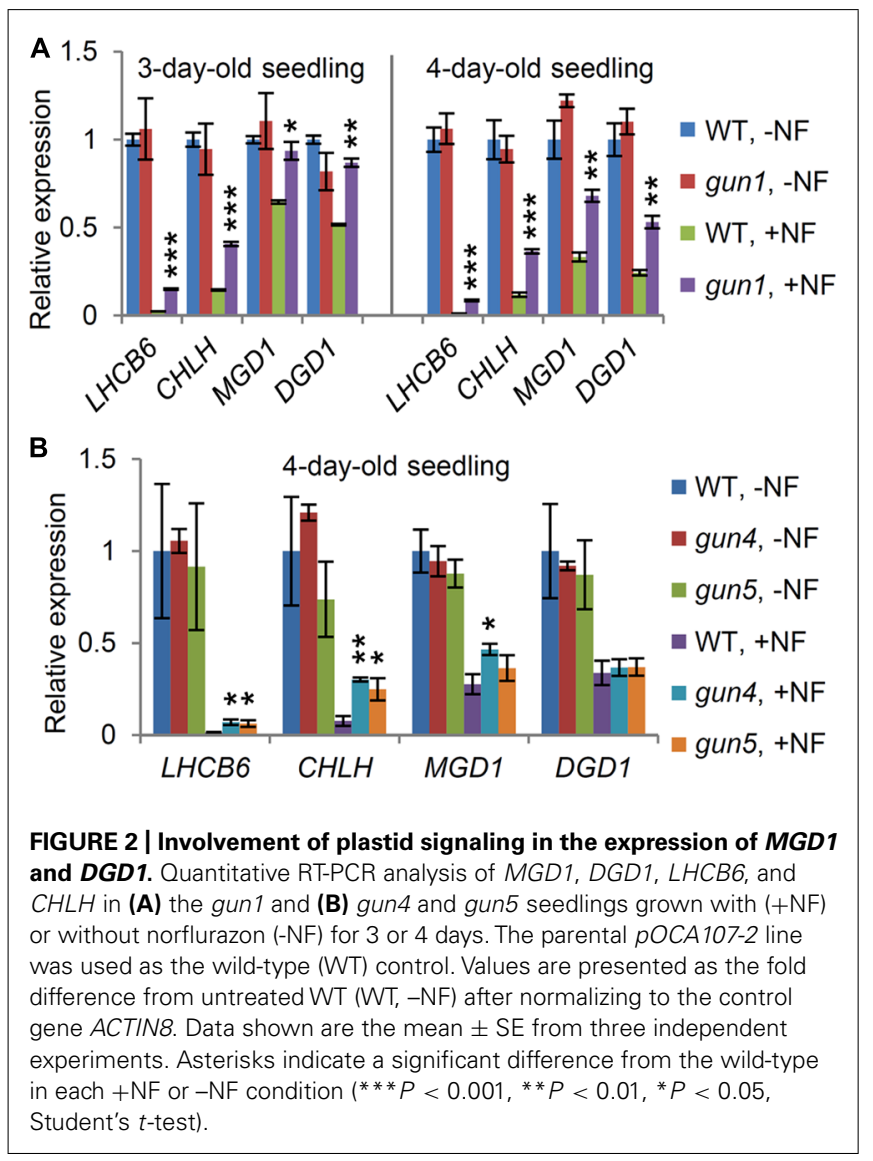

To ascertain a possible involvement of plastid signaling through the chlorophyll biosynthetic pathway in the downregulation of $M G D 1$ and DGD1, we investigated gun4 and gun5 mutants, which have a gun phenotype due to mutations in the GUN4 and the CHLH genes, respectively (Mochizuki et al., 2001; Larkin et al., 2003). In this experiment, we grew seedlings in the presence or absence of NF for 4 days after planting. In the absence of NF, both gun 4 and gun5 showed no decrease in MGD1 and DGD1 expression compared with the wild-type (Figure 2B), despite having reduced chlorophyll in the leaves (Mochizuki et al., 2001). The results agree with the data in Figure 1, showing that a slight decrease in chlorophyll synthesis does not influence the expression of the galactolipid synthesis genes. In the presence of NF, however, LHCB6 and CHLH expression in the gun 4 and gun5 mutants was substantially higher than that in the wild-type control, consistent with previous reports (Strand et al., 2003; Moulin et al., 2008). Meanwhile, unlike in gun1, the expression levels of MGD1 and DGD1 in the gun4 and gun5 mutants were not largely different from those in the wild-type, reflecting a minor impact of chlorophyll metabolism-mediated plastid signaling on the downregulation of the galactolipid synthesis genes.

\section{TRANSCRIPTIONAL REGULATION OF GALACTOLIPID SYNTHESIS BY LIGHT AND CYTOKININ DURING PHOTOMORPHOGENESIS}

Light plays a pivotal role in triggering chloroplast biogenesis, including galactolipid and chlorophyll synthesis during photomorphogenesis. We reported previously that MGD1, but not 


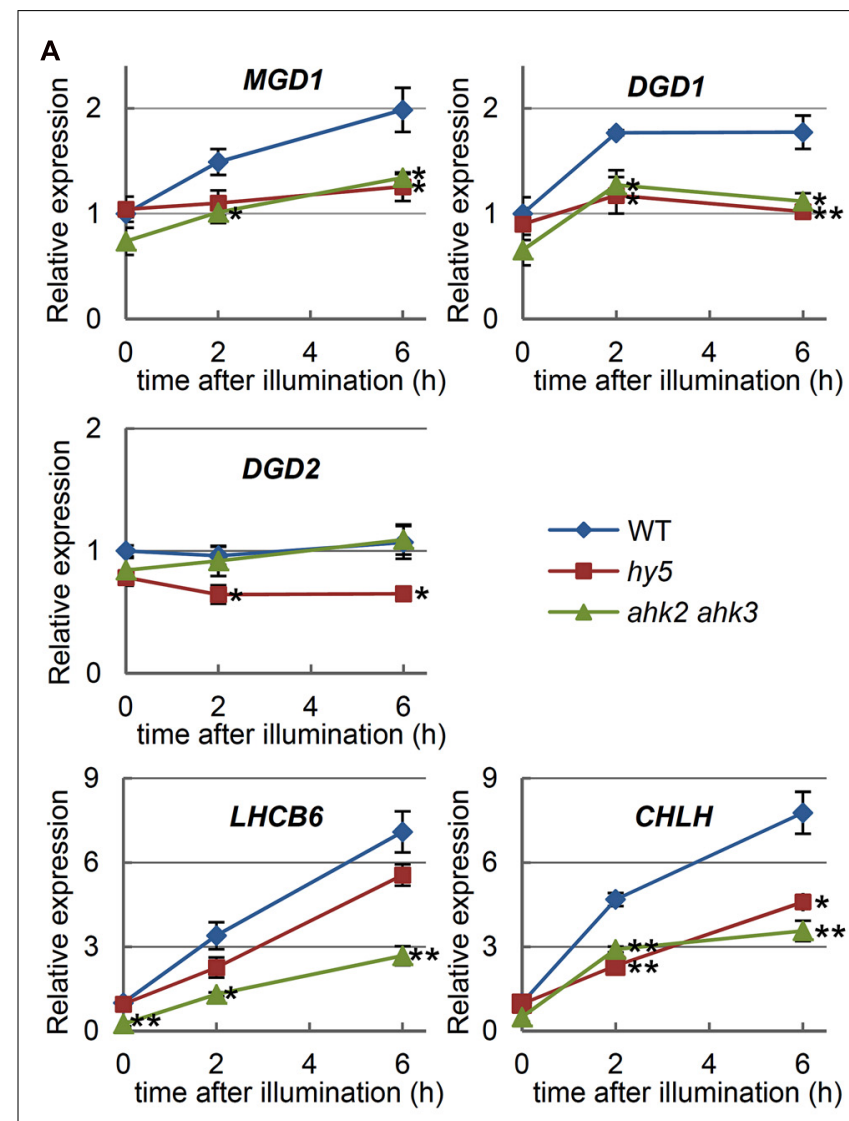

B

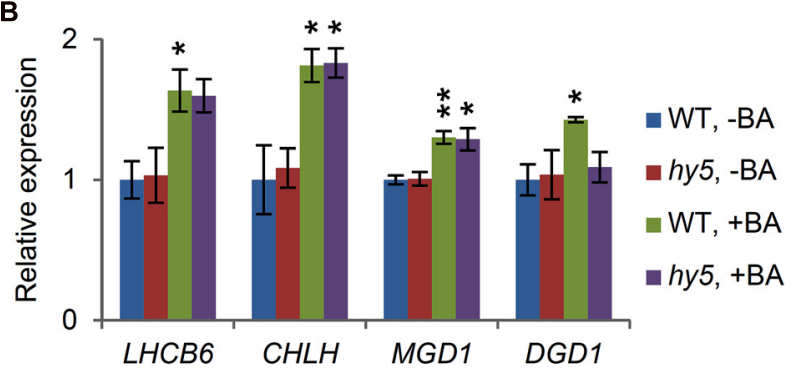

FIGURE 3 | Involvement of light and cytokinin signaling in the expression of MGD1 and DGD1. (A) Quantitative RT-PCR analysis of galactolipid synthesis genes (MGD1, DGD1, and DGD2) and

chlorophyll-related genes (LHCB6 and CHLH) in wild-type (WT), the hy5 mutant and the ahk2 ahk3 double mutant after illumination of 4-day-old etiolated seedlings for the indicated times. (B) Quantitative RT-PCR analysis in 4-day-old etiolated seedlings of WT and the hy5 mutant treated with 6-benzyladenine (+BA) or without BA (-BA) for $6 \mathrm{~h}$. Values are presented as the fold difference relative to $(\mathbf{A})$ the unilluminated seedlings $(0 \mathrm{~h})$ or $(\mathbf{B})$ the untreated wild-type control (WT, -BA) after normalizing to the control gene ACTIN8. Data shown are the mean $\pm \mathrm{SE}$ from three independent experiments. Asterisks indicate a significant difference (A) from the wild-type at each time point and (B) from the BA-untreated samples of each plant $\left({ }^{*} P<0.01,{ }^{*} P<0.05\right.$, Student's $t$-test).

MGD2 or MGD3, is upregulated by light (Kobayashi et al., 2004). Consistent with these results, MGD1 expression in the wild-type increased twofold after illumination of etiolated seedlings for $6 \mathrm{~h}$ (Figure 3A). No light induction analysis has been reported for $D G D$ genes, although mutant analyses demonstrate that DGD1 is mainly responsible for DGDG synthesis in photosynthetic tissues (Kelly et al., 2003). Investigation of the light responses of the DGD genes in the wild-type seedlings showed that $D G D 1$ expression had increased upon illumination, whereas $D G D 2$ expression remained unchanged (Figure 3A). This result supports the major and minor roles of $D G D 1$ and $D G D 2$, respectively, for thylakoid biogenesis during the development of photosynthetic tissues (Kelly et al., 2003). Light-induced upregulation of $L H C B 6$ and $C H L H$ was also observed. The increases of $L H C B 6$ and $C H L H$ expression were more pronounced than those of $M G D 1$ and $D G D 1$, in line with the extremely large accumulation of chlorophyll compared with the smaller increase in galactolipids during photomorphogenesis (Poincelot, 1973; Selstam and Sandelius, 1984).

It has been reported that transcription factor HY5, which is a positive regulator of photomorphogenesis downstream of multiple photoreceptors, directly targets many of the light-inducible photosynthesis-associated genes, including key chlorophyll synthesis genes (Lee et al., 2007). Indeed, upon illumination, the upregulation of $\mathrm{CHLH}$, which is a target of HY5, was strongly reduced in a null hy5 mutant (hy5-215; Figure 3A). In contrast, a deficiency of HY5 only weakly influenced LHCB6 expression, consistent with reports that $L H C B 6$ may not be a direct target of HY5 (Lee et al., 2007). It is interesting to note that among the galactolipid synthesis genes, $D G D 1$ and $D G D 2$ are putative direct targets of HY5 (Lee et al., 2007). In fact, the expression of these genes under light conditions was reduced in the hy 5 seedlings compared with the wild-type (Figure 3A). Moreover, we observed that light-induced upregulation of $M G D 1$ did not occur in $h y 5$, even though MGD1 was not perceived as a HY5 target. Our data suggest that HY5 plays a role in the light-induction of genes involved in galactolipid and chlorophyll synthesis, both directly and indirectly.

In addition to light, cytokinin has been reported to stimulate expression of MGD1 in etiolated seedlings (Kobayashi et al., 2004). To assess the involvement of cytokinin signaling in the light-response of galactolipid synthesis genes, a double mutant for cytokinin receptors (ahk2 ahk3; Higuchi et al., 2004) was investigated. Under dark conditions, the expression of both MGD1 and DGD1 decreased by $\sim 30 \%$ in ahk 2 ahk 3 compared with the wild-type, although the differences were not significant (Figure 3A). The expression of $L H C B 6$ and $C H L H$ under dark conditions also decreased in ahk2 ahk3. The data may reflect a minor role for cytokinin signaling in the expression of these genes during skotomorphogenesis. Furthermore, light induction of MGD1 and DGD1 expression did not occur in the double mutant. A similar result was observed for $\mathrm{LHCB} 6$ and $\mathrm{CHLH}$ expression, whereas DGD2 expression was not different between the wild-type or the ahk2 ahk3 mutants under light conditions. Our data demonstrate that cytokinin signaling is involved in a coordinated upregulation of the light-responsive genes during photomorphogenesis.

Exogenous cytokinin treatment is known to induce chloroplast development and to activate light-regulated promoters under dark conditions (Chory et al., 1994). In our experiments, expression of $L H C B 6$ and $C H L H$ under dark conditions was significantly upregulated after treatment with $1 \mu \mathrm{M} \mathrm{BA}$, a synthetic cytokinin (Figure 3B), which is consistent with the findings that cytokinin 
stimulates chlorophyll synthesis in etiolated seedlings (Masuda et al., 1995; Hedtke et al., 2012). As reported previously (Kobayashi et al., 2004), MGD1 expression was also significantly upregulated by BA treatment under dark conditions. In addition, DGD1 expression increased in response to $\mathrm{BA}$, supporting the results obtained from the ahk2 ahk3 mutant that cytokinin signaling has a positive effect on the expression of these genes. Since it has been reported that cytokinin can promote the expression of anthocyanin biosynthetic genes through stabilization of the HY5 protein (Vandenbussche et al., 2007), we investigated the involvement of HY5 in cytokinin signaling. When the hy5 seedlings were treated with $1 \mu \mathrm{M}$ BA under dark conditions, MGD1 was upregulated to almost the same level as that in the BA-treated wild-type seedlings (Figure 3B). LHCB6 and CHLH expression in the BA-treated hy5 seedlings was also equivalent to the BA-treated wild-type seedlings, suggesting that BA can upregulate these genes independently of HY5. In contrast, the increase in DGD1 expression by BA appeared to be canceled in hy5. Thus, the regulatory mechanisms may differ between MGD1 and $D G D 1$, as represented by the direct and non-direct targeting by HY5.

\section{HOMEOSTATIC TRANSCRIPTIONAL REGULATION OF GALACTOLIPID BIOSYNTHESIS}

Genes for the major galactolipid synthesis pathway (MGD1 and $D G D 1)$, but not those for the alternative pathway (MGD2, $M G D 3$, and DGD2), are upregulated along with photosynthesisassociated genes in response to light (Figure 3A; Kobayashi et al., 2004), consistent with the crucial roles of the major pathway in thylakoid biogenesis and photosynthesis (Kobayashi et al., 2009b). To assess the importance of light and cytokinin signaling pathways for homeostatic gene expression in galactolipid synthesis during early seedling growth, we analyzed the expression of MGD1 and DGD1 and that of LHCB6 and CHLH in the hy5 and ahk2 ahk3 mutants, which were grown for 7 days under continuous white light. Although the expression of these genes appeared to decrease in the mutants compared with the wild-type, only the reduction in DGD1 expression in ahk2 ahk3 was statistically significant (Figure 4A), suggesting a reduced importance of HY5 and cytokinin signaling in the homeostatic expression of these genes under continuous light conditions.

We assessed whether other factors associated with chloroplast biogenesis were involved in the regulation of the galactolipid synthesis genes. In this study, we examined the involvement of two types of transcription factors, namely GATA factors (GNC and CGA1) and GLK factors (GLK1 and GLK2), in the expression of MGD1 and DGD1. In the 7-day-old gnc cgal double mutant, the expression of both $\mathrm{LHCB} 6$ and $\mathrm{CHLH}$ was less than half that in the wild-type (Figure 4A), consistent with the reduced chlorophyll contents in mutants deficient in these factors in various tissues and conditions (Hudson et al., 2011; Chiang et al., 2012). DGD1 expression levels also decreased significantly in the mutant, whereas the decrease in MGD1 expression was not statistically significant. These data indicate that GNC and CGA1 are involved in the expression of the galactolipid synthesis genes, but that their influences are marginal.

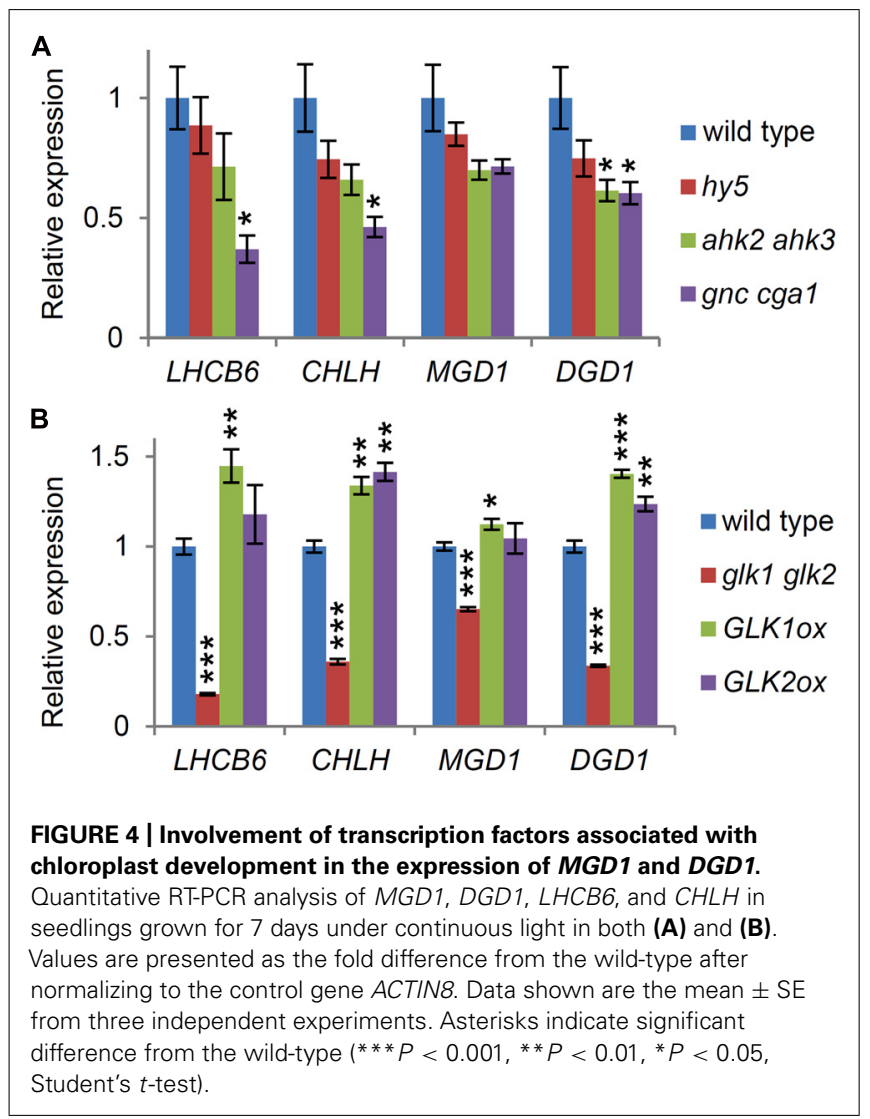

Next we analyzed the expression levels of these genes in a double mutant ( $g l k 1 g l k 2)$ and in overexpressors of the GLK factors (GLK1ox and GLK2ox). As reported previously (Waters et al., 2009), the expression of LHCB6 and CHLH, known targets of GLKs, decreased markedly in the $g l k 1$ glk2 mutant, whereas their expression levels increased slightly in the GLK overexpressors (Figure 4B). Interestingly, DGD1 showed an expression pattern similar to that of $L H C B 6$ and $C H L H$ in these plants, although DGD1 was not a reported target of the GLK factors (Waters et al., 2009). MGD1 expression also decreased significantly in the $g l k 1$ glk2 mutant, but the decrease was less than those of other genes examined. Furthermore, no significant upregulation of MGD1 was observed in either GLK1ox or GLK2ox, indicating a minor involvement of GLK factors in the regulation of MGD1 expression compared with DGD1 expression. As discussed later, smaller reductions in $M G D 1$ expression than those in $D G D 1$ in these mutants may reflect differences in mode of regulation for each step; DGD1 is subject to strong transcriptional regulation, whereas MGD1 may be rather under posttranscriptional regulation.

\section{REGULATION OF GALACTOLIPID METABOLISM DURING CHLOROPLAST DIFFERENTIATION IN THE ROOT}

We recently reported that chloroplast development in the roots is regulated through auxin/cytokinin signaling, involving at least two transcription factors, HY5 and GLK2 (Kobayashi et al., 2012a). In fact, seedlings treated with BA, or auxin-signaling mutants, like the slr-1 mutant, accumulated chlorophyll in the roots and displayed 


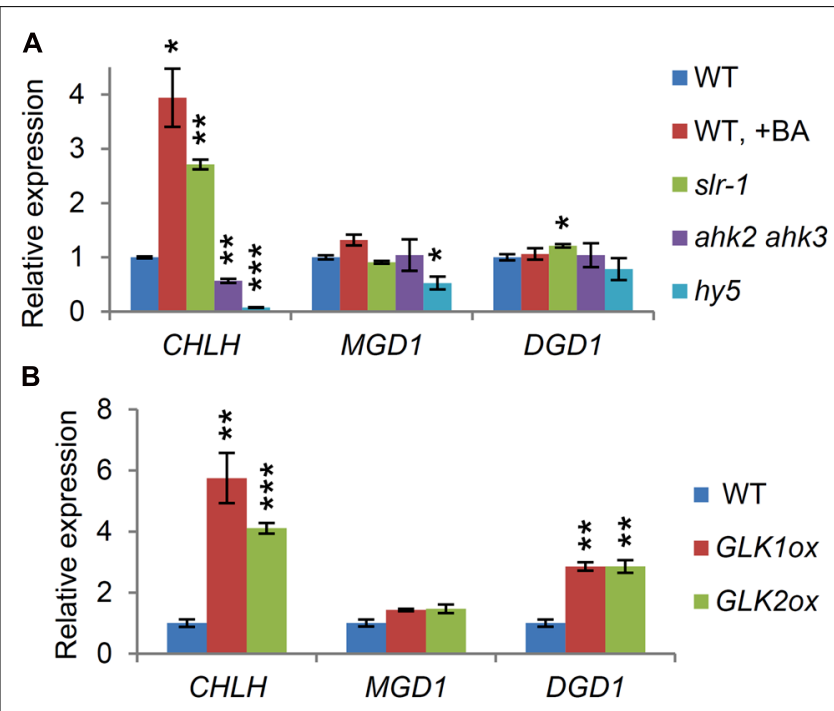

FIGURE 5 | Regulation of MGD1 and DGD1 expression in the root. Quantitative RT-PCR analysis of CHLH, MGD1 and DGD1 in roots of (A) 14-day-old seedlings and (B) 21-day-old seedlings grown under continuous white light. Values are presented as the fold difference from the untreated wild-type control (WT) after normalizing to the control gene ACTIN8. Data shown are the mean $\pm \mathrm{SE}$ from three independent experiments. +BA, 6-benzyladenine treatment for 7 days. Asterisks indicate significant difference from the wild-type control ${ }^{* * *} P<0.001,{ }^{*} P<0.01$, ${ }^{*} P<0.05$, Student's $t$-test).

an upregulation in chlorophyll synthesis genes, such as $\mathrm{CHLH}$ (Kobayashi etal., 2012a). Meanwhile, the roots of the hy5 and the ahk2 ahk3 double mutant showed reductions in chlorophyll synthesis gene expression and chlorophyll accumulation. Because the expression of MGD1 and DGD1 had a pattern similar to that of $C H L H$ during photomorphogenesis (Figure 3), we examined whether $M G D 1$ and $D G D 1$ were upregulated together with $C H L H$ during chloroplast differentiation in the roots. Whereas $\mathrm{CHLH}$ expression in the roots increased in the $s l r-1$ mutant and the BAtreated wild-type and decreased in $h y 5$ and $a h k 2 a h k 3$ as reported previously (Kobayashi et al., 2012a), the expression of MGD1 and $D G D 1$ in these root samples remained at levels similar to that in the untreated wild-type, except for some slight, but significant, changes (Figure 5A). This result indicates an uncoordinated regulation of gene expression between chlorophyll synthesis and galactolipid synthesis during chloroplast development in the root.

We recently reported that GLK factors strongly induce chloroplast development with an upregulation of photosynthesisassociated genes in the root (Kobayashi et al., 2012a, 2013b). To address whether the excessive chlorophyll synthesis accompanied upregulation of galactolipid synthesis genes in the roots, we examined the expression levels of MGD1 and DGD1 in the roots of the GLK overexpressors. The results showed that DGD1, but not $M G D 1$, was strongly upregulated together with CHLH in GLK1ox and GLK2ox roots (Figure 5B).

To assess whether MGD1 and DGD1 expression was coupled with lipid homeostasis during chloroplast differentiation, we compared galactolipid contents in the roots between the wild-type and GLK1ox. In the GLK1ox root, the proportions of MGDG and DGDG to total membrane glycerolipids increased more than twofold compared with those in the wild-type root (Figure 6A), showing that GLK1-mediated chloroplast differentiation accompanies the accumulation of galactolipids without upregulation of $M G D 1$ expression in the root. When the fatty acid composition of MGDG in the GLK1ox root was compared with that in the wildtype root, the proportions of triunsaturated fatty acids (16:3 and 18:3) increased in the GLK1ox root (Figure 6B). The increase in the 18:3 fatty acids was also observed in DGDG of the GLK1ox root, suggesting activation of a leaf-type galactolipid metabolism in the root plastids (Kobayashi et al., 2009b). The accumulation of galactolipids is consistent with the prominent development of thylakoid membrane networks within the plastids in the GLK1ox root (Kobayashi etal., 2013b). Because fatty acid desaturation in plastids requires ferredoxins as electron donors and is closely linked with the photosynthetic activities (Shanklin and Cahoon, 1998), development of thylakoid membranes and photosynthetic machineries may affect desaturase activities in plastids and thereby alter fatty acid compositions of galactolipids in GLK1ox roots. It is also possible that GLK1ox upregulates genes involved in fatty acid desaturation in roots.

\section{DISCUSSION}

\section{TRANSCRIPTIONAL COORDINATION OF GALACTOLIPID SYNTHESIS WITH CHLOROPHYLL SYNTHESIS AND CHLOROPLAST DEVELOPMENT}

In our previous study, we reported that galactolipid biosynthetic activities profoundly influence gene expression related to photosynthesis and chlorophyll synthesis; a lack of galactolipid synthesis in the mgd1-2 mutant caused a marked downregulation of the photosynthesis-associated genes, whereas partial complementation of galactolipid synthesis in $m g d 1-2$ by the alternative MGD2/MGD3 pathway attenuated the downregulation of these genes (Kobayashi et al., 2013a). In this study, we

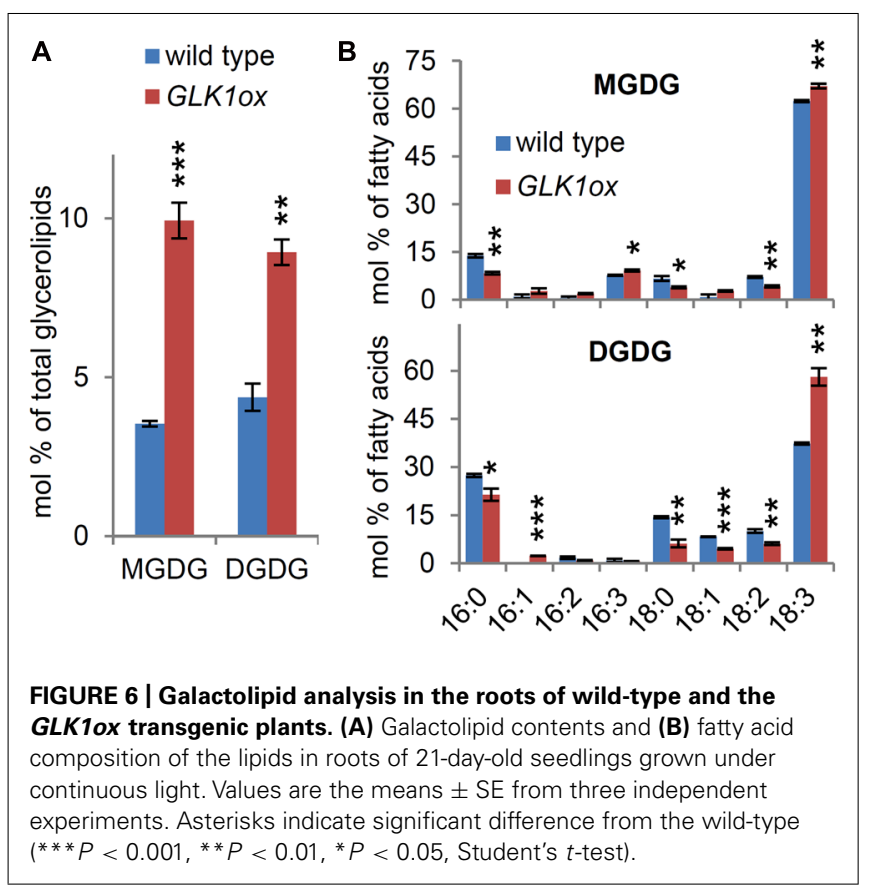


revealed that tetrapyrrole metabolism also affected the expression of galactolipid synthesis genes, as observed by the downregulation of MGD1 and DGD1 in the chlh and hemal mutants (Figure 1B). Taken together, our data suggest a strong transcriptional link between galactolipid and chlorophyll synthesis, which would be required for the coordinated formation of lipid bilayers, with chlorophyll synthesis and the assembly of photosynthetic complexes during thylakoid biogenesis. One possible mechanism that coordinates the expression of the galactolipid synthesis genes with that of the photosynthesisassociated genes is plastid signaling. Indeed, as with many photosynthesis-associated genes, MGD1 and DGD1 expression was regulated by plastid signaling, in which GUN1 played a pivotal role (Figure 2A). It is possible that in chlh and hema1, impairments in chloroplast function due to disruption of tetrapyrrole metabolism, induce plastid signaling via GUN1, which downregulates the galactolipid synthesis genes. It is interesting to note that MGD1 and DGD1 expression was not repressed in the chli1 mutant, although the chlorophyll deficiency in chlil was almost the same as in hemal (Figure 1), suggesting that the inability to accumulate chlorophyll does not simply trigger downregulation of the galactolipid synthesis genes. It has recently been reported that increased flux through the heme branch of the tetrapyrrole biosynthetic pathway restores the expression of photosynthesis-associated nuclear genes under chloroplast-defective conditions, suggesting that heme synthesized in plastids may be used as a retrograde signal to upregulate nuclear photosynthetic genes (Woodson et al., 2011). Thus, it is possible that changes in heme metabolism would also contribute to the downregulation of the galactolipid synthesis genes in hemal. In the chlh mutant, the complete lack of chlorophyll synthesis may largely disrupt tetrapyrrole metabolism or lead to secondary effects in chloroplast functioning that induce downregulation of the galactolipid synthesis genes. On the other hand, in the chli1 mutant, the CHLI2 isoform can partially complement the loss of CHLI1 in the chelation of Mg to protoporphyrin IX (Kobayashi et al., 2008; Huang and Li, 2009) and thus the remaining activity by CHLI2 might maintain MGD1 and DGD1 expression at wild-type levels in chli1.

\section{COEXPRESSION OF THE MAJOR GALACTOLIPID SYNTHESIS GENES WITH PHOTOSYNTHESIS-ASSOCIATED NUCLEAR GENES}

When chloroplasts are differentiated from proplastids or etioplasts under light conditions, large amounts of galactolipids are synthesized, together with chlorophylls and photosynthetic proteins, to form functional thylakoid membrane networks. Consistent with the bulk of galactolipids in thylakoid membranes being synthesized via the MGD1-DGD1 pathway (Kobayashi et al., 2009b), the expression of both MGD1 and DGD1 was induced by light during photomorphogenesis. In leaves, the expression patterns of these galactolipid synthesis genes were generally similar to those of $L H C B 6$ and $C H L H$ in various mutants and under various growth conditions, although the amplitude of the expression changes were different (Figures 2-4), suggesting the existence of co-regulatory mechanisms between the galactolipid synthesis genes and the chlorophyll-related genes. It has been reported that key chlorophyll synthesis genes, including $\mathrm{CHLH}$, form tight coexpression networks with other photosynthesis-associated nuclear genes, like LHCB6, suggesting that the regulatory coexpression in the nucleus plays a central role in the assembly of the photosynthetic machinery during chloroplast biogenesis (Kobayashi et al., 2012b). In our results, MGD1 and DGD1 showed a pattern of coexpression with $\mathrm{LHCB} 6$ and $\mathrm{CHLH}$; however, these galactolipid synthesis genes are not found in the coexpression networks of the photosynthesis-associated genes. Because galactolipids are predominant not only in the thylakoid membranes of chloroplasts but also in the envelope membranes of plastids (Block et al., 1983), it is reasonable to assume that the expression profiles of the galactolipid synthesis genes differ from those of the photosynthetic genes, particularly in non-photosynthetic organs, such as the roots (Figure 5). Furthermore, additional unique functions other than photosynthesis have been proposed for galactolipids under several growth conditions, including phosphate starvation (Kobayashi et al., 2009b), wounding stress (Nakashima et al., 2013), and cold (Moellering et al., 2010). In fact, MGD1 expression is highly upregulated by salt and drought stress in roots and by wounding in leaves (Kobayashi et al., 2009b), whereas DGD1 expression is upregulated in response to limitations in phosphate and nitrogen (Gaude et al., 2007). This unique regulation of MGD1 and DGD1 may result in the removal of the galactolipid synthesis genes from photosynthetic gene coexpression lists that have been constructed from the large quantity of Arabidopsis transcriptomic data collected to date (ATTED-II, http://atted.jp/).

\section{TRANSCRIPTIONAL FACTORS INVOLVED IN THE REGULATION OF THE GALACTOLIPID SYNTHESIS GENES AND CHLOROPHYLL SYNTHESIS GENES}

We have shown that light signaling via HY5 and cytokinin signaling via AHK2 and AHK3 are involved in upregulation of MGD1 and DGD1 during photomorphogenesis (Figure 3A). A similar result was observed for $\mathrm{CHLH}$ expression, whereas only cytokinin signaling had any significant effect on $L H C B 6$ expression. Genome-wide analysis has identified DGD1 and CHLH as direct targets of HY5 (Lee et al., 2007), and therefore, HY5 may directly upregulate these genes in response to illumination. Because cytokinin is reported to have a stabilizing effect on the HY5 protein (Vandenbussche et al., 2007), it is possible that reduced cytokinin signaling in the ahk2 ahk3 double mutant led to a decrease in HY5 activity and, thereby, inhibited the upregulation of DGD1 and CHLH. This assumption is consistent with the absence of a BA-induced increase in DGD1 expression in the hy5 mutant (Figure 3B), suggesting a pivotal role for HY5 in DGD1 expression during photomorphogenesis. However, BA-induced upregulation of $\mathrm{CHLH}$ was not repressed in the hy5 mutant. Moreover, LHCB6 expression, which is only mildly affected by HY5, also decreased in the ahk2 ahk3 mutant and increased after BA treatment. These results show that cytokinin signaling can act on photosynthetic gene expression, independently of HY5. In the case of MGD1, reduced MGD1 expression in the ahk2 ahk3 mutant during photomorphogenesis agrees with similar results in cucumbers, in which cytokinins are essential for the upregulation of cucumber MGD1 during photomorphogenesis (Yamaryo et al., 2003). In addition, 
the upregulation of MGD1 in response to light was attenuated in the hy5 mutant, even though MGD1 was not identified as a HY5 target (Lee et al., 2007). Because the HY5 protein is subject to degradation via an E3-ubiquitin ligase CONSTITUTIVE PHOTOMORPHOGENIC1 (COP1) in the dark (Osterlund et al., 2000), it is possible that COP1 downregulates MGD1 along with other HY5-regulated genes in the dark through degradation of HY5. HY5 is also required for MGD1 expression in illuminated roots (Figure 5A). Considering that COP1 functions in suppressing chloroplast development in roots of light-grown Arabidopsis (Deng and Quail, 1992), the COP1-HY5 signaling may also be involved in the regulation of MGD1 in roots. There has been no in-depth analysis of the MGD1 promoter region, and therefore we cannot exclude the possibility of direct regulation of MGD1 by HY5. Alternatively, it is possible that a transcription factor(s) involved in MGD1 expression requires HY5 to function during photomorphogenesis.

In the 7-day-old seedlings, the expression of MGD1 and DGD1 decreased in the $g l k 1$ glk2 double mutant to a greater extent than in the gnc cgal double mutant (Figure 4), suggesting a stronger impact of the GLK factors on the expression of these genes, as observed in the expression of LHCB6 and CHLH, which are proposed to be direct targets of the GLK factors (Waters et al., 2009). Unlike LHCB6 and CHLH, MGD1 expression decreased only slightly in the $g l k 1$ glk 2 mutant and did not increase in the GLK overexpressors, consistent with MGD1 not being the target of the GLK factors (Waters etal., 2009). Because key genes involved in tetrapyrrole synthesis, including HEMA1 and CHLH, are strongly downregulated in $g l k 1$ glk2, together with a reduction in chlorophyll contents (Fitter et al., 2002; Waters et al., 2009), it is possible that impairment of tetrapyrrole synthesis would cause the decrease in MGD1 expression in $g l k 1$ $g l k 2$, as in the hemal and chlh mutants (Figure 1B). Meanwhile, the expression profiles of DGD1 in $g l k 1$ glk2 and in the overexpressors, were very similar to those of LHCB6 and CHLH, even though DGD1 has not been identified as a GLK target (Waters et al., 2009). DGD1 expression also increased in the GLK1ox root (Figure 5B). These data suggest that GLK factors act as pivotal regulators of DGD1, although the mode of action of GLKs in the regulation of DGD1 remains to be elucidated.

\section{DISTINCT REGULATION OF GALACTOLIPID SYNTHESIS AND CHLOROPHYLL SYNTHESIS DURING CHLOROPLAST BIOGENESIS IN ROOTS}

We reported that chlorophyll synthesis in roots is regulated by auxin/cytokinin signaling at the transcriptional level via the combined action of HY5 and the GLK factors (Kobayashi et al., 2012a). Although the expression of MGD1 and DGD1 changed in tandem with $L H C B 6$ and $C H L H$ during leaf development (Figures 2-4), MGD1 and DGD1 expression in the root differed substantially from $\mathrm{CHLH}$ expression (Figure 5A), showing the uncoupled transcriptional regulation of chlorophyll and galactolipid synthesis during chloroplast biogenesis in the root. Because galactolipids are constitutively required for plastid envelopes, even in non-photosynthetic roots, whereas the requirements of chlorophylls in roots are limited and changeable in response to the growth environment, the transcriptional regulatory systems may differ between these two metabolic processes in non-photosynthetic organs.

In a previous report, we have shown that galactolipids accumulate in the root of $s l r-1$ mutants without upregulation of MGD2, MGD3, DGD1, and DGD2 under nutrient-sufficient conditions (Narise et al., 2010). Furthermore, MGD1 expression in the slr-1 root remained at the same level as in the wild-type plants (Figure 5A), indicating that the increase in galactolipids in the $s l r-1$ roots was not due to the transcriptional activation of the galactolipid synthesis genes. We did not see a marked upregulation of MGD1 in the GLK1ox root, despite the elevated accumulation of MGDG (Figure 5B). It has been reported that plant MGDG synthases are subject to two types of post-translational regulation: the activation by thioredoxins in a redox-dependent manner (Yamaryo et al., 2006; Shimojima et al., 2013) and the activation by anionic phospholipids, phosphatidic acid, and phosphatidylglycerol (Dubots et al., 2010; Shimojima et al., 2013). Therefore, the post-translational activation of MGDG synthesis may contribute to the elevated accumulation of MGDG in the greenish roots. Because it is proposed that the enzymatic regulation of MGDG synthesis activity is not indispensable but is important for the proper development of thylakoid membranes in Arabidopsis (Masuda et al., 2011), the post-translational regulation of MGDG synthesis could play a role in coordinating galactolipid synthesis with the formation of photosynthetic machineries, in combination with transcriptional regulation. Several distinct expression patterns between MGD1 and DGD1 that were observed in the responsiveness to chloroplast dysfunctions and to the changes in the transcriptional regulators may reflect differences in the involvement of post-transcriptional regulation. This may be the case because DGD1 expression was more flexible in response to changes in chloroplast development, compared with MGD1 expression. It is likely that DGDG synthesis by DGD1 is more strongly regulated at the transcription level than is MGDG synthesis by MGD1.

\section{ACKNOWLEDGMENTS}

For the supply of Arabidopsis lines, we thank Nobuyoshi Mochizuki, Kyoto University (cs, chli1, chlh, hema1, gun1-1, gun41, and gun5-1), Jane A. Langdale, Oxford University ( $g l k 1$ glk2, GLK1ox, and GLK2ox), Tatsuo Kakimoto, Osaka University ( $a h k 2-$ 2 ahk3-3), Kiyotaka Okada, National Institute for Basic Biology (hy5-215), Hidehiro Fukaki, Kobe University ( $s l r-1)$, and Eric G. Schaller ( gnc cga1). This work was supported by Grants-inAid for Scientific Research on Priority Areas (Nos. 24770055 and 24570042).

\section{REFERENCES}

Awai, K., Maréchal, E., Block, M. A., Brun, D., Masuda, T., Shimada, H., et al. (2001). Two types of MGDG synthase genes, found widely in both 16:3 and 18:3 plants, differentially mediate galactolipid syntheses in photosynthetic and nonphotosynthetic tissues in Arabidopsis thaliana. Proc. Natl. Acad. Sci. U.S.A. 98, 10960-10965. doi: 10.1073/pnas.181331498

Block, M. A., Dorne, A. J., Joyard, J., and Douce, R. (1983). Preparation and characterization of membrane fractions enriched in outer and 
inner envelope membranes from spinach chloroplasts. II. Biochemical characterization. J. Biol. Chem. 258, 13281-13286. doi: 10.1007/978-94-0174973-2_5

Chiang, Y. H., Zubo, Y. O., Tapken, W., Kim, H. J., Lavanway, A. M., Howard, L., et al. (2012). Functional characterization of the GATA transcription factors GNC and CGA1 reveals their key role in chloroplast development, growth, and division in Arabidopsis. Plant Physiol. 160, 332-348. doi: 10.1104/pp.112.198705

Chory, J., Reinecke, D., Sim, S., Washburn, T., and Brenner, M. (1994). A role for cytokinins in de-etiolation in Arabidopsis (det mutants have an altered response to cytokinins). Plant Physiol. 104, 339-347.

Deng, X. W., and Quail, P. H. (1992). Genetic and phenotypic characterization of cop1 mutants of Arabidopsis thaliana. Plant J. 2, 83-95. doi: 10.1111/j.1365313X.1992.00083.x

Dörmann, P., Hoffmann-Benning, S., Balbo, I., and Benning, C. (1995). Isolation and characterization of an Arabidopsis mutant deficient in the thylakoid lipid digalactosyl diacylglycerol. Plant Cell 7, 1801-1810. doi: 10.1105/tpc.7.11.1801

Dubots, E., Audry, M., Yamaryo, Y., Bastien, O., Ohta, H., Breton, C., et al. (2010). Activation of the chloroplast monogalactosyldiacylglycerol synthase MGD1 by phosphatidic acid and phosphatidylglycerol. J. Biol. Chem. 285, 6003-6011. doi: 10.1074/jbc.M109.071928

Fitter, D. W., Martin, D. J., Copley, M. J., Scotland, R. W., and Langdale, J. A. (2002). GLK gene pairs regulate chloroplast development in diverse plant species. Plant J. 31, 713-727. doi: 10.1046/j.1365-313X.2002.01390.x

Fukaki, H., Tameda, S., Masuda, H., and Tasaka, M. (2002). Lateral root formation is blocked by a gain-of-function mutation in the SOLITARY-ROOT/IAA14 gene of Arabidopsis. Plant J. 29, 153-168. doi: 10.1046/j.0960-7412.2001. 01201.x

Gaude, N., Bréhélin, C., Tischendorf, G., Kessler, F., and Dörmann, P. (2007). Nitrogen deficiency in Arabidopsis affects galactolipid composition and gene expression and results in accumulation of fatty acid phytyl esters. Plant J. 49, 729-739. doi: 10.1111/j.1365-313X.2006.02992.x

Gaude, N., Tippmann, H., Flemetakis, E., Katinakis, P., Udvardi, M., and Dörmann, P. (2004). The galactolipid digalactosyldiacylglycerol accumulates in the peribacteroid membrane of nitrogen-fixing nodules of soybean and lotus. J. Biol. Chem. 279, 34624-34630. doi: 10.1074/jbc.M404098200

Hedtke, B., Alawady, A., Albacete, A., Kobayashi, K., Melzer, M., Roitsch, T., et al. (2012). Deficiency in riboflavin biosynthesis affects tetrapyrrole biosynthesis in etiolated Arabidopsis tissue. Plant Mol. Biol. 78, 77-93. doi: 10.1007/s11103-0119846-1

Higuchi, M., Pischke, M. S., Mähönen, A. P., Miyawaki, K., Hashimoto, Y., Seki, M., et al. (2004). In planta functions of the Arabidopsis cytokinin receptor family. Proc. Natl. Acad. Sci. U.S.A. 101, 8821-8826. doi: 10.1073/pnas. 0402887101

Huang, Y. S., and Li, H. M. (2009). Arabidopsis CHLI2 can substitute for CHLI1. Plant Physiol. 150, 636-645. doi: 10.1104/pp.109.135368

Hudson, D., Guevara, D., Yaish, M. W., Hannam, C., Long, N., Clarke, J. D., et al. (2011). GNC and CGA1 modulate chlorophyll biosynthesis and glutamate synthase (GLU1/Fd-GOGAT) expression in Arabidopsis. PLoS ONE 6:e26765. doi: 10.1371/journal.pone.0026765

Jarvis, P., and López-Juez, E. (2013). Biogenesis and homeostasis of chloroplasts and other plastids. Nat. Rev. Mol. Cell Biol. 14, 787-802. doi: 10.1038/nrm3702

Kelly, A. A., and Dörmann, P. (2002). DGD2, an Arabidopsis gene encoding a UDP-galactose-dependent digalactosyldiacylglycerol synthase is expressed during growth under phosphate-limiting conditions. J. Biol. Chem. 277, 1166-1173. doi: 10.1074/jbc.M110066200

Kelly, A. A., Froehlich, J. E., and Dörmann, P. (2003). Disruption of the two digalactosyldiacylglycerol synthase genes DGD1 and DGD2 in Arabidopsis reveals the existence of an additional enzyme of galactolipid synthesis. Plant Cell 15, 2694-2706. doi: 10.1105/tpc016675

Kobayashi, K., Awai, K., Nakamura, M., Nagatani, A., Masuda, T., and Ohta, H. (2009a). Type-B monogalactosyldiacylglycerol synthases are involved in phosphate starvation-induced lipid remodeling, and are crucial for lowphosphate adaptation. Plant J. 57, 322-331. doi: 10.1111/j.1365-313X.2008. 03692.x

Kobayashi, K., Awai, K., Takamiya, K., and Ohta, H. (2004). Arabidopsis type B monogalactosyldiacylglycerol synthase genes are expressed during pollen tube growth and induced by phosphate starvation. Plant Physiol. 134, 640-648. doi: 10.1104/pp.103.032656
Kobayashi, K., Baba, S., Obayashi, T., Sato, M., Toyooka, K., Keränen, M., et al. (2012a). Regulation of root greening by light and auxin/cytokinin signaling in Arabidopsis. Plant Cell 24, 1081-1095. doi: 10.1105/tpc.111. 092254

Kobayashi, K., Kondo, M., Fukuda, H., Nishimura, M., and Ohta, H. (2007). Galactolipid synthesis in chloroplast inner envelope is essential for proper thylakoid biogenesis, photosynthesis, and embryogenesis. Proc. Natl. Acad. Sci. U.S.A. 104, 17216-17221. doi: 10.1073/pnas.0704680104

Kobayashi, K., Mochizuki, N., Yoshimura, N., Motohashi, K., Hisabori, T., and Masuda, T. (2008). Functional analysis of Arabidopsis thaliana isoforms of the Mg-chelatase CHLI subunit. Photochem. Photobiol. Sci. 7, 1188-1195. doi: $10.1039 / \mathrm{b} 802604 \mathrm{c}$

Kobayashi, K., Nakamura, Y., and Ohta, H. (2009b). Type A and type B monogalactosyldiacylglycerol synthases are spatially and functionally separated in the plastids of higher plants. Plant Physiol. Biochem. 47, 518-525. doi: 10.1016/j.plaphy.2008.12.012

Kobayashi, K., Narise, T., Sonoike, K., Hashimoto, H., Sato, N., Kondo, M., etal. (2013a). Role of galactolipid biosynthesis in coordinated development of photosynthetic complexes and thylakoid membranes during chloroplast biogenesis in Arabidopsis. Plant J. 73, 250-261. doi: 10.1111/ tpj. 12028

Kobayashi, K., Obayashi, T., and Masuda, T. (2012b). Role of the G-box element in regulation of chlorophyll biosynthesis in Arabidopsis roots. Plant Signal. Behav. 7, 922-926. doi: 10.4161/psb.20760

Kobayashi, K., Sasaki, D., Noguchi, K., Fujinuma, D., Komatsu, H., Kobayashi, M., et al. (2013b). Photosynthesis of root chloroplasts developed in Arabidopsis lines overexpressing GOLDEN2-LIKE transcription factors. Plant Cell Physiol. 54, 1365-1377. doi: 10.1093/pcp/pct086

Koncz, C., Mayerhofer, R., Koncz-Kalman, Z., Nawrath, C., Reiss, B., Redei, G. P., etal. (1990). Isolation of a gene encoding a novel chloroplast protein by T-DNA tagging in Arabidopsis thaliana. EMBO J. 9, 1337-1346.

Larkin, R. M., Alonso, J. M., Ecker, J. R., and Chory, J. (2003). GUN4, a regulator of chlorophyll synthesis and intracellular signaling. Science 299, 902-906. doi: 10.1126/science. 1079978

Lee, J., He, K., Stolc, V., Lee, H., Figueroa, P., Gao, Y., et al. (2007). Analysis of transcription factor HY5 genomic binding sites revealed its hierarchical role in light regulation of development. Plant Cell 19, 731-749. doi: 10.1105/tpc.106. 047688

Masuda, S., Harada, J., Yokono, M., Yuzawa, Y., Shimojima, M., Murofushi, K., et al. (2011). A monogalactosyldiacylglycerol synthase found in the green sulfur bacterium Chlorobaculum tepidum reveals important roles for galactolipids in photosynthesis. Plant Cell 23, 2644-2658. doi: 10.1105/tpc.111.085357

Masuda, T., Ohta, H., Shioi, Y., Tsuji, H., and Takamiya, K. (1995). Stimulation of glutamyl-tRNA reductase activity by benzyladenine in greening cucumber cotyledons. Plant Cell Physiol. 36, 1237-1243.

Melis, A., Spangfort, M., and Andersson, B. (1987). Light-absorption and electron transport balance between photosystem-II and photosystem-I in spinach chloroplasts. Photochem. Photobiol. 45, 129-136. doi: 10.1111/j.17511097.1987.tb08413.x

Mochizuki, N., Brusslan, J. A., Larkin, R., Nagatani, A., and Chory, J. (2001). Arabidopsis genomes uncoupled 5 (GUN5) mutant reveals the involvement of $\mathrm{Mg}$-chelatase $\mathrm{H}$ subunit in plastid-to-nucleus signal transduction. Proc. Natl. Acad. Sci. U.S.A. 98, 2053-2058. doi: 10.1073/pnas.98.4.2053

Moellering, E. R., Muthan, B., and Benning, C. (2010). Freezing tolerance in plants requires lipid remodeling at the outer chloroplast membrane. Science 330, 226228. doi: 10.1126/science. 1191803

Moulin, M., McCormac, A. C., Terry, M. J., and Smith, A. G. (2008). Tetrapyrrole profiling in Arabidopsis seedlings reveals that retrograde plastid nuclear signaling is not due to Mg-protoporphyrin IX accumulation. Proc. Natl. Acad. Sci. U.S.A. 105, 15178-15183. doi: 10.1073/pnas.0803054105

Nakamura, H., Muramatsu, M., Hakata, M., Ueno, O., Nagamura, Y., Hirochika, H., et al. (2009). Ectopic overexpression of the transcription factor OsGLK1 induces chloroplast development in non-green rice cells. Plant Cell Physiol. 50, 1933-1949. doi: $10.1093 / \mathrm{pcp} / \mathrm{pcp} 138$

Nakashima, A., von Reuss, S. H., Tasaka, H., Nomura, M., Mochizuki, S., Iijima, Y., et al. (2013). Traumatin- and dinortraumatin-containing galactolipids in Arabidopsis: their formation in tissue-disrupted leaves as counterparts of 
green leaf volatiles. J. Biol. Chem. 288, 26078-26088. doi: 10.1074/jbc.M113. 487959

Narise, T., Kobayashi, K., Baba, S., Shimojima, M., Masuda, S., Fukaki, H., et al. (2010). Involvement of auxin signaling mediated by IAA14 and ARF7/19 in membrane lipid remodeling during phosphate starvation. Plant Mol. Biol. 72, 533-544. doi: 10.1007/s11103-009-9589-4

Osterlund, M. T., Hardtke, C. S., Wei, N., and Deng, X. W. (2000). Targeted destabilization of HY5 during light-regulated development of Arabidopsis. Nature 405, 462-466. doi: 10.1038/35013076

Oyama, T., Shimura, Y., and Okada, K. (1997). The Arabidopsis HY5 gene encodes a bZIP protein that regulates stimulus-induced development of root and hypocotyl. Genes Dev. 11, 2983-2995. doi: 10.1101/gad.11. 22.2983

Pfaffl, M. W. (2001). A new mathematical model for relative quantification in realtime RT-PCR. Nucleic Acids Res. 29:e45. doi: 10.1093/nar/29.9.e45

Poincelot, R. P. (1973). Differences in lipid composition between undifferentiated and mature maize chloroplasts. Plant Physiol. 51, 802-804. doi: 10.1104/pp.51.4.802

Powell, A. L., Nguyen, C. V., Hill, T., Cheng, K. L., Figueroa-Balderas, R., Aktas, H., et al. (2012). Uniform ripening encodes a Golden 2-like transcription factor regulating tomato fruit chloroplast development. Science 336, 1711-1715. doi: $10.1126 /$ science. 1222218

Rissler, H. M., Collakova, E., DellaPenna, D., Whelan, J., and Pogson, B. J. (2002). Chlorophyll biosynthesis. Expression of a second Chl I gene of magnesium chelatase in Arabidopsis supports only limited chlorophyll synthesis. Plant Physiol. 128, 770-779. doi: 10.1104/pp.010625

Selstam, E., and Sandelius, A. S. (1984). A comparison between prolamellar bodies and prothylakoid membranes of etioplasts of dark-grown wheat concerning lipid and polypeptide composition. Plant Physiol. 76, 1036-1040. doi: 10.1104/pp.76.4.1036

Shanklin, J., and Cahoon, E. B. (1998). Desaturation and related modifications of fatty acids. Annu. Rev. Plant Physiol. Plant Mol. Biol. 49, 611-641. doi: 10.1146/annurev.arplant.49.1.611

Shimojima, M., Watanabe, T., Madoka, Y., Koizumi, R., Yamamoto, M. P., Masuda, K., et al. (2013). Differential regulation of two types of monogalactosyldiacylglycerol synthase in membrane lipid remodeling under phosphate-limited conditions in sesame plants. Front. Plant Sci. 4:469. doi: 10.3389/fpls.2013. 00469

Strand, A., Asami, T., Alonso, J., Ecker, J. R., and Chory, J. (2003). Chloroplast to nucleus communication triggered by accumulation of $\mathrm{Mg}$-protoporphyrinIX. Nature 421, 79-83. doi: 10.1038/nature01204

Susek, R. E., Ausubel, F. M., and Chory, J. (1993). Signal transduction mutants of Arabidopsis uncouple nuclear $C A B$ and RBCS gene expression from chloroplast development. Cell 74, 787-799. doi: 10.1016/0092-8674(93) 90459-4
Tanaka, R., Kobayashi, K., and Masuda, T. (2011). Tetrapyrrole metabolism in Arabidopsis thaliana. Arabidopsis Book 9:e0145. doi: 10.1199/ tab.0145

Vandenbussche, F., Habricot, Y., Condiff, A. S., Maldiney, R., Van der Straeten, D., and Ahmad, M. (2007). HY5 is a point of convergence between cryptochrome and cytokinin signalling pathways in Arabidopsis thaliana. Plant J. 49, 428-441. doi: 10.1111/j.1365-313X.2006. 02973.x

Waters, M. T., Moylan, E. C., and Langdale, J. A. (2008). GLK transcription factors regulate chloroplast development in a cell-autonomous manner. Plant J. 56, 432444. doi: 10.1111/j.1365-313X.2008.03616.x

Waters, M. T., Wang, P., Korkaric, M., Capper, R. G., Saunders, N. J., and Langdale, J. A. (2009). GLK transcription factors coordinate expression of the photosynthetic apparatus in Arabidopsis. Plant Cell 21, 1109-1128. doi: 10.1105/tpc.108. 065250

Woodson, J. D., Perez-Ruiz, J. M., and Chory, J. (2011). Heme synthesis by plastid ferrochelatase I regulates nuclear gene expression in plants. Curr. Biol. 21, 897903. doi: 10.1016/j.cub.2011.04.004

Yamaryo, Y., Kanai, D., Awai, K., Shimojima, M., Masuda, T., Shimada, H., et al. (2003). Light and cytokinin play a co-operative role in MGDG synthesis in greening cucumber cotyledons. Plant Cell Physiol. 44, 844-855. doi: $10.1093 / \mathrm{pcp} / \mathrm{pcg} 110$

Yamaryo, Y., Motohashi, K., Takamiya, K., Hisabori, T., and Ohta, H. (2006). In vitro reconstitution of monogalactosyldiacylglycerol (MGDG) synthase regulation by thioredoxin. FEBS Lett. 580:4086-4090. doi: 10.1016/j.febslet.2006. 06.050

Conflict of Interest Statement: The authors declare that the research was conducted in the absence of any commercial or financial relationships that could be construed as a potential conflict of interest.

Received: 23 January 2014; accepted: 25 May 2014; published online: 11 June 2014. Citation: Kobayashi K, Fujii S, Sasaki D, Baba S, Ohta H, Masuda T and Wada H (2014) Transcriptional regulation of thylakoid galactolipid biosynthesis coordinated with chlorophyll biosynthesis during the development of chloroplasts in Arabidopsis. Front. Plant Sci. 5:272. doi: 10.3389/fpls.2014.00272

This article was submitted to Plant Systems Biology, a section of the journal Frontiers in Plant Science.

Copyright (c) 2014 Kobayashi, Fuiii, Sasaki, Baba, Ohta, Masuda and Wada. This is an open-access article distributed under the terms of the Creative Commons Attribution License (CC BY). The use, distribution or reproduction in other forums is permitted, provided the original author(s) or licensor are credited and that the original publication in this journal is cited, in accordance with accepted academic practice. No use, distribution or reproduction is permitted which does not comply with these terms. 